PROCEEDINGS OF THE

AMERICAN MATHEMATICAL SOCIETY

Volume 126, Number 6, June 1998, Pages 1627-1635

S $0002-9939(98) 04372-X$

\title{
ENVELOPING ALGEBRAS OF LIE COLOR ALGEBRAS: PRIMENESS VERSUS GRADED-PRIMENESS
}

\author{
JEFFREY BERGEN AND D. S. PASSMAN \\ (Communicated by Ken Goodearl)
}

\begin{abstract}
Let $G$ be a finite abelian group and let $L$ be a, possibly restricted, $G$-graded Lie color algebra. Then the enveloping algebra $U(L)$ is also $G$ graded, and we consider the question of whether $U(L)$ being graded-prime implies that it is prime. The first section of this paper is devoted to the special case of Lie superalgebras over a field $K$ of characteristic $\neq 2$. Specifically, we show that if $i=\sqrt{-1} \in K$ and if $U(L)$ has a unique minimal graded-prime ideal, then this ideal is necessarily prime. As will be apparent, the latter result follows quickly from the existence of an anti-automorphism of $U(L)$ whose square is the automorphism of the enveloping algebra associated with its $\mathbb{Z}_{2}$-grading. The second section, which is independent of the first, studies more general Lie color algebras and shows that if $U(L)$ is graded-prime and if most homogeneous components $L_{g}$ of $L$ are infinite dimensional over $K$, then $U(L)$ is prime. Here we use $\Delta$-methods to study the grading on the extended centroid $C$ of $U(L)$. In particular, if $G$ is generated by the infinite support of $L$, then we prove that $C=C_{1}$ is homogeneous.
\end{abstract}

\section{$\S 1$. Superalgebras}

Let $L=L_{0} \oplus L_{1}$ be an ordinary (or restricted) Lie superalgebra over any field $K$ of characteristic not 2, and let $U(L)$ denote its ordinary (or restricted) enveloping algebra. Then $U(L)=U_{0} \oplus U_{1}$ is $\mathbb{Z}_{2}$-graded, and we let $\sigma$ denote the corresponding automorphism of order 2 defined by $\sigma(u)=u$ if $u \in U_{0}$ and $\sigma(u)=-u$ if $u \in U_{1}$. Then it is clear that any subspace of $U(L)$ is graded if and only if it is $\sigma$-stable. In particular, an ideal $I \triangleleft U(L)$ is graded-prime if and only if it is $\sigma$-prime. The goal of this section is to show that if $i=\sqrt{-1} \in K$, then $U(L)$ is a graded-prime ring if and only if it is a prime ring. Of course, prime always implies graded-prime, so we are essentially concerned with the reverse implication.

Lemma 1.1. Let $U(L)$ denote the (restricted) enveloping algebra of the (restricted) $K$-superalgebra $L$ and assume that $i=\sqrt{-1} \in K$. If $\tau: U(L) \rightarrow U(L)$ is given by $\tau(x)=-x$ if $x \in L_{0}$ and $\tau(x)=i x$ if $x \in L_{1}$, then $\tau$ is a well-defined algebra anti-automorphism of $U(L)$ and $\tau^{2}=\sigma$.

Received by the editors November 22, 1996

1991 Mathematics Subject Classification. Primary 16S30, 16W55, 17 B35.

The first author's research was supported by the Faculty Research and Development Fund of the College of Liberal Arts \& Sciences at DePaul University. The second author's research was supported in part by NSF Grant DMS-9622566.

(C)1998 American Mathematical Society 
Proof. Consider the map $\theta: L \rightarrow U(L)^{\text {op }}$ given by $\theta(x)=-x^{\text {op }}$ if $x \in L_{0}$ and $\theta(x)=i x^{\text {op }}$ if $x \in L_{1}$. We show that $\theta$ is a Lie superalgebra homomorphism into the superalgebra of the $\mathbb{Z}_{2}$-graded ring $U(L)^{\mathrm{op}}$. To avoid confusion, let us use [[, ]] to denote the bracket defined by the latter algebra. Let $x \in L_{0}$ and let $y \in L_{a}$ with $a \in\{0,1\}$. Then $[x, y] \in L_{a}$, so $\theta(y)=\lambda y^{\mathrm{op}}$ and $\theta([x, y])=\lambda[x, y]^{\text {op }}$ for the same scalar $\lambda$. Thus

$$
\begin{aligned}
{[[\theta(x), \theta(y)]] } & =\left[\left[-x^{\mathrm{op}}, \lambda y^{\mathrm{op}}\right]\right]=-\lambda\left(x^{\mathrm{op}} y^{\mathrm{op}}-y^{\mathrm{op}} x^{\mathrm{op}}\right)=-\lambda(y x-x y)^{\mathrm{op}} \\
& =\lambda(x y-y x)^{\mathrm{op}}=\lambda[x, y]^{\mathrm{op}}=\theta([x, y]) .
\end{aligned}
$$

Similarly, if $x, y \in L_{1}$, then $[x, y] \in L_{0}$, so

$$
\begin{aligned}
{[[\theta(x), \theta(y)]] } & =\left[\left[i x^{\mathrm{op}}, i y^{\mathrm{op}}\right]\right]=i^{2}\left(x^{\mathrm{op}} y^{\mathrm{op}}+y^{\mathrm{op}} x^{\mathrm{op}}\right)=-(y x+x y)^{\mathrm{op}} \\
& =-(x y+y x)^{\mathrm{op}}=-[x, y]^{\mathrm{op}}=\theta([x, y]) .
\end{aligned}
$$

In particular, if $L$ is an ordinary Lie superalgebra, then the universal property of $U(L)$ implies that $\theta$ extends to an algebra homomorphism $\theta: U(L) \rightarrow U(L)^{\mathrm{op}}$. By composing this with the natural algebra anti-isomorphism $U(L)^{\text {op }} \rightarrow U(L)$, we conclude that $\tau$ exists and is an algebra anti-homomorphism. Since $\tau^{2}$ agrees with $\sigma$ on $L$, we conclude that $\tau^{2}=\sigma$ and hence that $\tau$ is an algebra anti-automorphism of order dividing 4.

Finally, if $L$ is a restricted Lie superalgebra in characteristic $p>2$, then the above argument will apply provided we show that $\theta$ also respects the $p$ th power map ${ }^{[p]}: L_{0} \rightarrow L_{0}$. To this end, let $x \in L_{0}$ and note that $x^{p}=x^{[p]}$ in the restricted enveloping algebra $U(L)$. Thus

$$
\theta\left(x^{[p]}\right)=-\left(x^{[p]}\right)^{\mathrm{op}}=-\left(x^{p}\right)^{\mathrm{op}}=\left(-x^{\mathrm{op}}\right)^{p}=\theta(x)^{p},
$$

and $\theta$ extends to an algebra homomorphism $\theta: U(L) \rightarrow U(L)^{\mathrm{op}}$, as required.

We remark that any anti-automorphism $\tau$ of a ring $R$ permutes its ideals, preserving inclusion, and maps primes to primes. Furthermore, if $\tau$ commutes with an automorphism $\sigma$, then $\tau$ permutes the $\sigma$-stable ideals of $R$ and hence it maps $\sigma$-primes to $\sigma$-primes. With this observation, we have

Theorem 1.2. Let $L$ be a (restricted) Lie superalgebra over the field $K$ of characteristic $\neq 2$, let $U(L)$ denote its (restricted) enveloping algebra, and assume that $i=\sqrt{-1} \in K$. If $U(L)$ has a unique minimal graded-prime ideal $P$, then $P$ is the unique minimal prime of $U(L)$.

Proof. Let $\sigma$ be the automorphism of $U(L)$ of order 2 associated with the grading and, since $i=\sqrt{-1} \in K$, let $\tau$ be defined as in the preceding lemma. Then $\tau$ commutes with $\tau^{2}=\sigma$, so $\tau$ permutes the graded-prime ideals of $U(L)$ and hence $\tau$ stabilizes $P$, the unique minimal graded-prime. In particular, $\tau$ gives rise to an anti-automorphism of the $\sigma$-prime ring $R=U(L) / P$. Since $\sigma$ is an automorphism of $R$ of order $\leq 2$, [P, page 133] implies that $R$ has at most two minimal primes, say $Q$ and $Q^{\sigma}$, and that $Q \cap Q^{\sigma}=0$. But then the cyclic group $\left\{1, \tau, \tau^{2}, \tau^{3}\right\}$ permutes these $\leq 2$ minimal primes, and hence $\tau^{2}=\sigma$ must act trivially as a permutation. Thus $Q=Q^{\sigma}$ and $0=Q \cap Q^{\sigma}=Q$. This means that $R$ is a prime ring and hence that $P$ is a prime ideal of $U(L)$. Finally, if $T$ is any prime ideal of $U(L)$, then $T \cap T^{\sigma}$ is $\sigma$-prime, so $T \cap T^{\sigma} \supseteq P$ and consequently $T \supseteq P$. Thus $P$ is the unique minimal prime of $U(L)$. 
Note that $[\mathrm{Bl}]$ asks whether the enveloping algebra $U(L)$ of a finite-dimensional Lie superalgebra $L$ must necessarily have a unique minimal prime. In view of the above, we can essentially obtain an affirmative answer here by showing that $U(L)$ has a unique minimal graded-prime ideal. For example, in the following result, we are able to slightly sharpen the conclusion of [L, Theorem I(i)] concerning minimal graded-prime ideals when $L$ is solvable.

Corollary 1.3. Let $L$ be a finite-dimensional solvable Lie superalgebra over a field $K$ of characteristic 0 . Then $U(L)$ has a unique minimal prime ideal.

Proof. If $F$ is the algebraic closure of $K$, then [L, Theorem I(i)] and the preceding result imply that $F \otimes U(L) \cong U(F \otimes L)$ has a unique minimal prime, say $Q$. In particular, since $F \otimes U(L)$ is Noetherian, it follows that $Q$ is nilpotent. Now $U(L)$ is also Noetherian, so there exist minimal primes $P_{1}, P_{2}, \ldots, P_{n}$ of $U(L)$, not necessarily distinct, with $P_{1} P_{2} \cdots P_{n}=0$. Lifting these to ideals of $F \otimes U(L)$, we have

$$
\left(F \otimes P_{1}\right)\left(F \otimes P_{2}\right) \cdots\left(F \otimes P_{n}\right)=0 \subseteq Q
$$

and therefore $Q \supseteq F \otimes P_{k} \supseteq P_{k}$ for some subscript $k$. But then $P_{k}$ is also nilpotent, and this easily implies that $P_{k}$ is the unique minimal prime of $U(L)$.

This, of course, extends [KK, Theorem 3.8] and indicates why the following result may be of interest.

Lemma 1.4. Let $L=L_{0} \oplus L_{1}$ be a finite-dimensional ordinary Lie superalgebra and assume that $U(L)$ has a unique minimal prime ideal. Then every non-nilpotent ideal of $U(L)$ has a nonzero intersection with $U\left(L_{0}\right)$.

Proof. If $T$ denotes the set of nonzero elements of $U\left(L_{0}\right)$, then clearly $T$ is a multiplicatively closed set of regular elements of $U(L)$. Furthermore, by [Bh, Section 4], $T$ is a right Ore set of $U(L)$ and $U(L) T^{-1}$ is the Artinian right classical quotient ring of the enveloping algebra. Now let $N$ denote the unique minimal prime ideal of $U(L)$, so that $N$ is also the unique largest nilpotent ideal of this Noetherian ring. If $I$ is a non-nilpotent ideal of $U(L)$, then $(I+N) / N$ is a nonzero ideal of the prime Noetherian ring $U(L) / N$, and hence $I$ contains an element $c$ which is regular modulo $N$. Indeed, [Sm, Theorem 2.12] implies that $c$ is regular in $U(L)$ and hence invertible in $U(L) T^{-1}$. Since the right ideal $I T^{-1}$ contains an invertible element, it follows that $1 \in I T^{-1}$, so $I \cap T \neq \emptyset$ and $I \cap U\left(L_{0}\right) \neq 0$.

Another consequence of Theorem 1.2 is a slight sharpening of [BP, Corollary 1.3]. If $L$ is a Lie superalgebra, recall that $\Delta_{L}$ is defined by

$$
\Delta_{L}=\left\{\ell \in L \mid \operatorname{dim}_{K} \ell \cdot \operatorname{ad} U(L)<\infty\right\} .
$$

Then [BP, Lemma 2.1] implies that $\Delta_{L}$ is a characteristic (restricted) Lie superideal of $L$ and that it is the join of all finite-dimensional superideals of $L$. The following is an immediate consequence of Theorem 1.2 and [BP, Corollary 1.3(ii)].

Corollary 1.5. Let $L$ be a Lie superalgebra over a field $K$ of characteristic $\neq 2$ and suppose that $i=\sqrt{-1} \in K$. If char $K=p>2$, assume further that $L$ is restricted. Then $U(L)$ is prime if and only if $U\left(\Delta_{L}\right)$ is graded $L$-prime. 
We use this to prove

Corollary 1.6. Let $L$ be a solvable Lie superalgebra over a field $K$ of characteristic 0 . Then $U(L)$ is prime if and only if it is semiprime.

Proof. Since prime always implies semiprime, we are concerned here with the reverse implication. Thus we suppose that $U(L)$ is semiprime, and we first consider the case where $i=\sqrt{-1} \in K$.

Suppose $A$ and $B$ are nonzero graded $L$-stable ideals of $U\left(\Delta_{L}\right)$ with $A B=0$. Since $\Delta_{L}$ is generated by the finite-dimensional superideals of $L$, there exists such an ideal $I$ of $L$ with $\tilde{A}=A \cap U(I) \neq 0$ and $\tilde{B}=B \cap U(I) \neq 0$. Now $I$ is finite dimensional and solvable, so Corollary 1.3 implies that the Noetherian ring $U(I)$ has a unique minimal prime which is nilpotent. In particular, since $\tilde{A} \tilde{B}=0$, we see that one of $\tilde{A}$ or $\tilde{B}$, say $\tilde{A}$, is nilpotent. But then $\tilde{A}$ is a nonzero graded $L$-stable nilpotent ideal of $U(I)$, so it is clear that $\tilde{A} U(L)$ is a nonzero nilpotent ideal of $U(L)$, a contradiction. It follows that $U\left(\Delta_{L}\right)$ is graded $L$-prime, and we conclude from the preceding corollary that $U(L)$ is prime.

Finally, let $K$ be an arbitrary field of characteristic 0 and set $F=K[i]$. Then $F / K$ is a finite Galois extension, so it follows that $F \otimes U(L)=U(F \otimes L)$ is also semiprime. Consequently, by the above, $F \otimes U(L)$ is prime, and hence so is $U(L)$.

Note that the preceding argument really only requires the fact that each finitedimensional superideal $I$ of $L$ is solvable. Indeed, we need only assume that, for each such $I, U(I)$ has a unique minimal prime ideal. We remark that if $H \triangleleft L$ and if $U(L)$ is semiprime, then it is not necessarily true that $U(H)$ is also semiprime. This occurs because superderivations need not stabilize minimal primes even if char $K=0$. For instance, we have

Example 1.7. If $K$ is any field of characteristic $\neq 2$, then there exists a metabelian (restricted) Lie superalgebra $L$ over $K$ such that $U(L)$ is prime while $U\left(\Delta_{L}\right)$ is not even semiprime.

Proof. Define $L=L_{0} \oplus L_{1}$ so that $L_{0}$ has $K$-basis $\{a, b\}$ and $L_{1}$ has $K$-basis $\left\{c, d_{0}, d_{1}, d_{2}, \ldots\right\}$. Furthermore, assume that $\left[c, d_{0}\right]=a,\left[b, d_{j}\right]=d_{j+1}$ for all $j \geq 0$, and that all other Lie brackets of basis elements are zero. Notice that $A=K a+K c$ and $B=K b+K d_{0}+K d_{1}+K d_{2}+\cdots$ are super-subalgebras of $L$ and that $L=A \rtimes B$. Indeed, $B^{\prime}=K b+K d_{1}+K d_{2}+\cdots$ is a superideal of $B$ and $B \rightarrow B / B^{\prime} \cong K d_{0} \rightarrow \operatorname{Der}(A)$ describes the action of $B$ on $A$. With this observation, it is clear that $L$ exists, and then $L$ is metabelian since $[L, L]=K a+K d_{1}+K d_{2}+\cdots$ is abelian.

Now $A \subseteq \Delta_{L}$ and $B$ has no nonzero finite-dimensional super ideals, so $A=\Delta_{L}$ and $U\left(\Delta_{L}\right)=K\left[a, c \mid c^{2}=0\right]$ is not semiprime. On the other hand, the $\mathbb{Z}_{2}$-grading of this algebra is given by $U\left(\Delta_{L}\right)=K[a] \oplus K[a] c$ and, since $\left[c, d_{0}\right]=a$, it follows that any nonzero graded $L$-stable ideal of this ring meets $K[a]$ nontrivially. In particular, $U\left(\Delta_{L}\right)$ is graded $L$-prime, and if we now suppose that $i=\sqrt{-1} \in K$, then Corollary 1.5 implies that $U(L)$ is prime when char $K=0$.

If char $K=p>2$, let $\tilde{L}$ be the $K$-subspace of $U(L)$ with $K$-basis given by $\left\{a^{p^{j}}, b^{p^{j}}, c, d_{j} \mid j \geq 0\right\}$. Then we know that $\tilde{L}$ is a restricted Lie superalgebra and that its restricted enveloping algebra $U(\tilde{L})$ is precisely equal to $U(L)$. Furthermore, 
$\Delta_{\tilde{L}}$ has $K$-basis $\left\{a, a^{p}, a^{p^{2}}, \ldots, c\right\}$, so $U\left(\Delta_{\tilde{L}}\right)=U\left(\Delta_{L}\right)=K\left[a, c \mid c^{2}=0\right]$ is graded $\tilde{L}$-prime. Thus, Corollary 1.5 again implies that $U(\tilde{L})=U(L)$ is prime.

Finally, if $K$ is arbitrary, set $F=K[i]$. Then we conclude from the preceding argument applied to $F \otimes L$ that $F \otimes U(L)=U(F \otimes L)$ is prime, and consequently $U(L)$ is also prime.

\section{$\S 2$. Color algebras}

Let $G$ be a finite abelian group and let $\epsilon: G \times G \rightarrow K^{\bullet}$ be a bicharacter. If $L$ is a Lie color algebra associated with $G$ and $\epsilon$, then $L=\oplus \sum_{g \in G} L_{g}$ is a $G$-graded $K$-vector space and there is a $K$-bilinear map [,] : $L \times L \rightarrow L$ which satisfies $\left[L_{x}, L_{y}\right] \subseteq L_{x y}$ for all $x, y \in G$. As usual, the support of $L$ is defined by

$$
\operatorname{supp} L=\left\{g \in G \mid L_{g} \neq 0\right\}
$$

and we can certainly assume that $G=\langle\operatorname{supp} L\rangle$ since the elements of $G$ outside of this supporting subgroup have no effect on the structure of $L$ or its enveloping algebra. Now each $g \in G$ gives rise to a linear character $\lambda_{g} \in \widehat{G}=\operatorname{Hom}\left(G, K^{\bullet}\right)$ defined by $\lambda_{g}(x)=\epsilon(g, x)$, and it is clear that the map $\Lambda: G \rightarrow \widehat{G}$ given by $g \mapsto \lambda_{g}$ is a group homomorphism. Again, we can assume that $\Lambda$ is one-to-one, since if $N=\operatorname{ker} \Lambda$ and $\bar{G}=G / N$, then $\epsilon$ determines a bicharacter $\bar{\epsilon}: \bar{G} \times \bar{G} \rightarrow K^{\bullet}$ and $L$ is naturally a Lie color algebra associated with $\bar{G}$ and $\bar{\epsilon}$. For convenience, we say that $G$ is minimal for $L$ if $G=\langle\operatorname{supp} L\rangle$ and $\Lambda: G \rightarrow \widehat{G}$ is one-to-one. In this case, it is clear that $G \cong \widehat{G}$.

Recall that $G$ is the disjoint union of the subsets $G_{+}=\{g \in G \mid \epsilon(g, g)=1\}$ and $G_{-}=\{g \in G \mid \epsilon(g, g)=-1\}$. Here $G_{+}$is a subgroup of $G$ of index $\leq 2$ and $G_{-}$ is the remaining coset when the index is 2 . If $L$ is an ordinary Lie superalgebra we let $U(L)$ denote its universal enveloping algebra, and we define the infinite support of $L$ by

$$
\operatorname{supp}_{\infty} L=\left\{g \in G_{+} \mid L_{g} \neq 0\right\} \cup\left\{g \in G_{-} \mid \operatorname{dim}_{K} L_{g}=\infty\right\} .
$$

Of course, $L$ is always an ordinary algebra when char $K=0$.

On the other hand, if char $K=p>0$, then $L$ may have the additional structure of a restricted color algebra. This means that there exists a $p$ th power map

$$
{ }^{[p]}: L_{g} \rightarrow L_{g^{p}} \quad \text { for all } g \in G_{+},
$$

and here we let $U(L)$ denote the restricted enveloping algebra of $L$. Furthermore, in this context the infinite support of $L$ is defined by

$$
\operatorname{supp}_{\infty} L=\left\{g \in G \mid \operatorname{dim}_{K} L_{g}=\infty\right\} .
$$

See $[\mathrm{BMPZ}]$ or $[\mathrm{S}]$ for a more complete description of Lie color algebras and their enveloping algebras.

Now let $R$ be any $K$-algebra and let $G$ be a finite abelian group with $G \cong$ $\widehat{G}=\operatorname{Hom}\left(G, K^{\bullet}\right)$. If $R=\oplus \sum_{g \in G} R_{g}$ is $G$-graded, then the dual group $\widehat{G}$ acts as automorphisms on $R$ by defining

$$
\left(\sum_{g \in G} r_{g}\right)^{\lambda}=\sum_{g \in G} \lambda(g) r_{g}
$$

for any $\lambda \in \widehat{G}$. Conversely, if $\widehat{G}$ acts on $R$, then $R$ is $G$-graded by the eigenspaces

$$
R_{g}=\left\{r \in R \mid r^{\lambda}=\lambda(g) r \text { for all } \lambda \in \widehat{G}\right\},
$$


which are indexed by the elements $g \in G$. Indeed, these are equivalent structures on $R$, since $G \cong \widehat{G}$ implies that for any two distinct elements $x, y \in G$ there exists $\lambda \in \widehat{G}$ with $\lambda(x) \neq \lambda(y)$.

Suppose, in addition, that $R$ is a semiprime $K$-algebra, and let $C=\mathcal{C}(R)$ denote its extended centroid (see $[\mathrm{A}]$ or $[\mathrm{M}]$ ). If $R$ is $G$-graded, then we know that the action of $\widehat{G}$ on $R$ extends naturally to an action on the central closure $R C$ of $R$. Hence the $G$-grading on $R$ extends to a grading on $R C$ with $C$ a graded subring. Note that the assumption $G \cong \widehat{G}$ holds when $R=U(L)$ and $G$ is minimal for $L$. Our goal in this section is to study the grading on the extended centroid of $U(L)$ when this enveloping algebra is semiprime, and following [W, Proposition 2.8] we define

$$
\Delta_{L}=\left\{\ell \in L \mid \operatorname{dim}_{K} \ell \cdot \operatorname{ad} U(L)<\infty\right\} .
$$

Then $\Delta_{L}$ is a characteristic (restricted) Lie color ideal of $L$ which "controls" the linear identities of $U(L)$. We use this fact to prove

Lemma 2.1. Assume that $R=U(L)$ is semiprime and let $c$ be a nonzero element of the extended centroid $C=\mathcal{C}(R)$. Then there exists a finite-dimensional graded subspace $V$ of $R$, which is stable under the adjoint action of $U(L)$ on $U(L)$, such that $0 \neq v c \in R$ for some $v \in V$.

Proof. Let us first assume that either char $K=0$, or char $K=p>0$ and that $L$ is restricted. If $I=\{r \in R \mid r c \in R\}$, then the definition of $C$ implies that $I$ is an ideal of $R$ with zero annihilator in $R$ and hence in $R C$. In particular, $I c \neq 0$ and we fix $\alpha \in I$ with $0 \neq \beta=\alpha c \in R$. Now let $r$ be any element of $I$ and set $s=r c \in R$. If $x \in R$, then we know that $x c=c x$ and, upon multiplying this equation on the left by $\alpha$ and on the right by $r$, we obtain

$$
\alpha x s=\beta x r \quad \text { for all } x \in R,
$$

a linear identity in $R$. In particular, since $\beta \neq 0$, this linear identity and [W, Theorem 4.8] imply that there exist elements $\alpha^{\prime}$ and $\beta^{\prime} \neq 0$ in $U\left(\Delta_{L}\right)$, depending only upon $\alpha$ and $\beta$, such that $\alpha^{\prime} s=\beta^{\prime} r$. But $s=r c=c r$, so this yields $\left(\alpha^{\prime} c-\beta^{\prime}\right) r=$ 0 and, since $r \in I$ is arbitrary, we have $\left(\alpha^{\prime} c-\beta^{\prime}\right) I=0$ and hence $\alpha^{\prime} c=\beta^{\prime}$.

Now, by definition of $\Delta_{L}$ and $U\left(\Delta_{L}\right)$, it follows that there exists a finitedimensional ad $U(L)$-stable graded subspace $H$ of $L$ such that $\alpha^{\prime} \in(K+H)^{n}$ for some integer $n \geq 0$. Thus $V=(K+H)^{n}$ is a finite-dimensional ad $U(L)$-stable graded subspace of $U(L)$, and $\alpha^{\prime}=v \in V$ satisfies $v c=\alpha^{\prime} c=\beta^{\prime} \in R \backslash 0$.

Finally, suppose that $L$ is an ordinary Lie color algebra in characteristic $p>0$ and let $\widetilde{L}$ be the restricted Lie color subalgebra of $U(L)$ generated by $L$. Then it is known that $U(\tilde{L})$, the restricted enveloping algebra of $\tilde{L}$, is precisely equal to $U(L)$. Thus, the result of the preceding paragraph applied to $U(\tilde{L})$ yields the corresponding result for $U(L)$.

We remark that if $c$ above is assumed to be homogeneous, then we can find an appropriate finite-dimensional graded subspace $V$ with $0 \neq V c \subseteq R$. This is essentially contained in the proof of the following result.

Theorem 2.2. Let $L$ be a (restricted) G-graded Lie color algebra over the field $K$ and assume that $G$ is minimal for $L$. Suppose that the (restricted) enveloping algebra $U(L)$ is semiprime and let $C$ denote its extended centroid. If $G$ is generated by the infinite support of $L$, then $C=C_{1}$ is homogeneous of grade $1 \in G$. 
Proof. Suppose, by way of contradiction, that $C_{x} \neq 0$ for some $1 \neq x \in G$ and choose $0 \neq c \in C_{x}$. By the previous lemma, let $V$ be a finite-dimensional graded subspace of $R=U(L)$, stable under the adjoint action of $U(L)$, such that $0 \neq$ $v c \in R$ for some $v \in V$. If $W=\{w \in V \mid w c \in R\}$, then, since $R$ is a graded subring of $R C$ and since $c$ is homogeneous, it follows that $W$ is a graded subspace of $V$. Furthermore, since $v \in W$, we see that $W c=\sum_{g \in G} W_{g} c$ is a nonzero finitedimensional graded subspace of $R$. Consequently, we can choose $\alpha \in W$ to be a homogeneous element such that $\operatorname{deg} \alpha c \geq \operatorname{deg} w c$ for all $w \in W$.

Since $G$ is minimal for $L$ and $x \neq 1$, we know that $1 \neq \lambda_{x} \in \widehat{G}$. In particular, ker $\lambda_{x}=\left\{g \in G \mid \lambda_{x}(g)=1\right\}$ is a proper subgroup of $G$ and hence it cannot contain all members of $\operatorname{supp}_{\infty} L$, which, by hypothesis, generates $G$. Thus there exists $y \in \operatorname{supp}_{\infty} L$ with $1 \neq \lambda_{x}(y)=\epsilon(x, y)$. Let $\ell$ be any element of $L_{y}$ and, for convenience, use ${ }^{\ell}$ to denote the adjoint action of $\ell$.

Since $\alpha$ is homogeneous, $c \in C_{x}$ and $\ell \in L_{y}$, we have $(\alpha c)^{\ell}=\alpha^{\ell} c+k^{\prime} \alpha c^{\ell}$ for some nonzero $k^{\prime} \in K$ depending upon the grades of $\alpha$ and $\ell$. Furthermore, since $c$ and $\ell$ commute, we have

$$
c^{\ell}=\ell c-\epsilon(x, y) c \ell=(1-\epsilon(x, y)) c \ell=k^{\prime \prime} c \ell,
$$

where $k^{\prime \prime}=1-\epsilon(x, y)$ is a nonzero element of $K$ since $\epsilon(x, y)=\lambda_{x}(y) \neq 1$. Thus, setting $k=-k^{\prime} k^{\prime \prime} \in K \backslash 0$, we have

$$
\alpha^{\ell} c=(\alpha c)^{\ell}-k^{\prime} \alpha c^{\ell}=(\alpha c)^{\ell}+k(\alpha c) \ell \in R .
$$

In particular, since $\alpha^{\ell} \in V^{\ell} \subseteq V$, we have $\alpha^{\ell} \in W$. Now $\operatorname{deg}(\alpha c)^{\ell} \leq \operatorname{deg} \alpha c$ is always true and $\operatorname{deg} \alpha^{\ell} c \leq \operatorname{deg} \alpha c$ follows from the maximality of $\operatorname{deg} \alpha c$. Thus the equation $k(\alpha c) \ell=\alpha^{\ell} c-(\alpha c)^{\ell}$ implies that $\operatorname{deg}(\alpha c) \ell \leq \operatorname{deg} \alpha c$ for all $\ell \in L_{y}$.

Since $\alpha c$ is a nonzero element of $U(L)$, there exists a finite-dimensional graded subspace $H$ of $L$ with $\alpha c \in(K+H)^{n}$ for some $n \geq 0$. In particular, if $\operatorname{dim}_{K} L_{y}=$ $\infty$, we can choose $\ell \in L_{y} \backslash H$, and consequently the PBW theorem implies that $\operatorname{deg}(\alpha c) \ell=1+\operatorname{deg} \alpha c>\operatorname{deg} \alpha c$, a contradiction. On the other hand, if $\operatorname{dim}_{K} L_{y}$ is finite, then the definition of $\operatorname{supp}_{\infty} L$ implies that $L$ is an ordinary Lie superalgebra and that $y \in G_{+}$. In this case, we know that $L_{y} \neq 0$ and, if $0 \neq \ell \in L_{y}$, then the PBW theorem again yields $\operatorname{deg}(\alpha c) \ell=1+\operatorname{deg} \alpha c>\operatorname{deg} \alpha c$, a contradiction. Thus the assumption that $C_{x} \neq 0$ for some $1 \neq x \in G$ is false, and the theorem is proved.

The preceding argument actually yields information on the allowable support of $C$ when $G$ is not necessarily generated by $\operatorname{supp}_{\infty} L$. Specifically, it shows that if $x \in$ $G$ satisfies $1 \neq \lambda_{x}\left(\operatorname{supp}_{\infty} L\right)=\epsilon\left(x, \operatorname{supp}_{\infty} L\right)$, then the homogeneous component $C_{x}$ must be zero.

Again, let $R$ be a $G$-graded $K$-algebra with $G \cong \widehat{G}$. Then any subspace $V$ of $R$ is $\widehat{G}$-stable if and only if it is $G$-graded. In particular, $R$ is $\widehat{G}$-prime if and only if it is graded-prime. Furthermore, when this occurs, then $R$ is $\widehat{G}$-semiprime and hence also semiprime since $\widehat{G}$ is finite. The following observation is well-known.

Lemma 2.3. Let $R$ be a $G$-graded $K$-algebra with $G \cong \widehat{G}$, and assume that $R$ is graded-prime. If $I$ is a nonzero ideal of $R$, then there exist a homogeneous element $a \in R$ and an element $c \in C=\mathcal{C}(R)$ with $0 \neq a c \in I$.

Proof. By assumption, $R=\oplus \sum_{g \in G} R_{g}$ is $G$-graded, and we can choose $0 \neq \alpha=$ $\sum_{g \in S} \alpha_{g} \in I$ with support $S \subseteq G$ and with $|S|$ minimal. Fix $h \in S$ and let $x \in R_{w}$ 
for some $w \in G$. Then

$$
\beta=\alpha_{h} x \alpha-\alpha x \alpha_{h}=\sum_{g \in S}\left(\alpha_{h} x \alpha_{g}-\alpha_{g} x \alpha_{h}\right) \in I,
$$

and note that $\alpha_{h} x \alpha_{g}-\alpha_{g} x \alpha_{h} \in R_{h w g}$ since $G$ is commutative. Furthermore, the $g=h$ summand vanishes here, and thus $\beta \in I$ has support contained in $h w(S \backslash\{h\})$, a set of size smaller than $|S|$. Thus $\beta=0$, and indeed

$$
\alpha_{h} x \alpha_{g}-\alpha_{g} x \alpha_{h}=0
$$

for all $g \in S$ and all $x \in R_{w}$. Since $R=\sum_{w \in G} R_{w}$, the above identity clearly holds for all $x \in R$.

Finally, note that the ideals $R \alpha_{h} R$ and $R \alpha_{g} R$ are nonzero and graded, and hence they have trivial annihilators in $R$ since $R$ is graded-prime. With this observation, it follows from the linear identity that, for each $g \in S$, there exists $c(g) \in C$ with $\alpha_{g}=\alpha_{h} c(g)$. (See, for example, the proof of [M, Theorem 1].) Thus

$$
\alpha=\sum_{g \in S} \alpha_{g}=\alpha_{h} \sum_{g \in S} c(g)=a c
$$

is a nonzero element of $I$ of the required form.

As a consequence, we have

Theorem 2.4. Let $L$ be a (restricted) G-graded Lie color algebra over the field $K$ and assume that $G$ is minimal for $L$. If the (restricted) enveloping algebra $U(L)$ is graded-prime and $G=\left\langle\operatorname{supp}_{\infty} L\right\rangle$, then $U(L)$ is prime.

Proof. As we observed, $R=U(L)$ is semiprime since it is graded-prime, and thus we can let $C=\mathcal{C}(R)$ denote its extended centroid. Furthermore, since $G=\left\langle\operatorname{supp}_{\infty} L\right\rangle$, Theorem 2.2 implies that $C=C_{1}$ is homogeneous. Now let $I$ be any nonzero ideal of $R$. Then the preceding lemma implies that there exist a homogeneous element $a \in R$ and an element $c \in C$ with $0 \neq a c \in I$. But $C=C_{1}$, so $c$ is also homogeneous and $a c$ is a nonzero homogeneous element of $I$. Thus $I$ contains the nonzero graded ideal $R a c R$ which has zero annihilator in the graded-prime ring $R$. It follows that $I$ also has zero annihilator in $R$, and therefore $R=U(L)$ is a prime ring.

We remark that some hypothesis is needed in the preceding theorem, since graded-prime enveloping algebras are not necessarily prime. Indeed, we mention

Example 2.5 $([\operatorname{Pr}])$. For any field $K$ of characteristic $\neq 2$ there exists a Lie color algebra $L$, graded by the fours group, such that $U(L)$ is a commutative algebra which is graded-prime but not prime.

Proof. Let $G=\{1, x, y, x y\} \cong\{1, x\} \times\{1, y\}$ be the fours group and let $K$ be a field of characteristic $\neq 2$. If $\epsilon: G \times G \rightarrow K^{\bullet}$ is the product of the super-bicharacters defined on $\{1, x\}$ and $\{1, y\}$, then $\epsilon(x, x)=\epsilon(y, y)=-1$ and $\epsilon(x, y)=1$. Now let $L$ be the $G$-graded Lie color algebra with $K$-basis $\{a, b, c\}$ such that $L_{x}=K a$, $L_{y}=K b, L_{1}=K c$ and $L_{x y}=0$. Furthermore, suppose that $[a, a]=[b, b]=2 c$ and that all other Lie products among the basis elements are 0 . Then it is easy to check that $L$ is indeed a Lie color algebra. Moreover, $U(L)$ is generated by $a, b, c$ with $a^{2}=b^{2}=c$ and $a b=b a$, since $[a, b]=0$ and $\epsilon(x, y)=1$. Thus the PBW theorem implies that $R=U(L)=K\left[a, b, c \mid a^{2}=b^{2}=c\right]$. Clearly $R$ is a commutative ring, which is not prime since $a \neq \pm b$ but $0=a^{2}-b^{2}=(a-b)(a+b)$. On the other hand, the grading of $R$ is given by $R_{1}=K[c], R_{x}=K[c] a, R_{y}=K[c] b$ and 
$R_{x y}=K[c] a b$. Consequently, each nonzero homogeneous element of $R$ is regular in $R$, and hence $R$ is graded-prime.

Note that the above mentioned ring is an ordinary enveloping algebra. However, when char $K=p>2$, then the same ring $R$ is also the restricted enveloping algebra of the restricted Lie color algebra $\tilde{L}$ with $K$-basis $\left\{a, b, c, c^{p}, c^{p^{2}}, \ldots\right\}$. Thus there also exist restricted enveloping algebras which are graded-prime but not prime.

Finally, Theorem 2.4 allows us to slightly sharpen [W, Theorem 5.4(ii)] when $G$ is generated by the infinite support of $L$. Specifically, we have

Corollary 2.6. Let $L$ be a G-graded Lie color algebra over the field $K$, and assume that $G$ is minimal for $L$ and that $G=\left\langle\operatorname{supp}_{\infty} L\right\rangle$. Furthermore, let $U(L)$ denote the enveloping algebra of $L$ if char $K=0$ or the restricted enveloping algebra of the restricted color algebra $L$ if $\operatorname{char} K=p>0$. Then $U(L)$ is prime if and only if $U\left(\Delta_{L}\right)$ is graded L-prime.

\section{REFERENCES}

[A] S. A. Amitsur, On rings of quotients, Symposia Math. 8 (1972), 149-164. MR 48:11180

[BMPZ] Y. Bahturin, A. Mikhalev, V. Petrogradsky, and M. Zaicev, Infinite Dimensional Lie Superalgebras, Walter de Gruyter, Berlin, New York, 1992. MR 94b:17001

[BP] J. Bergen and D. S. Passman, Delta methods in enveloping algebras of Lie superalgebras II, J. Algebra 166 (1994), 568-610. MR 95j:17007

[Bh] E. J. Behr, Enveloping algebras of Lie superalgebras, Pacific J. Math. 130 (1987), 9-25. MR 89b: 17023

[Bl] A. D. Bell, A criterion for primeness of enveloping algebras of Lie superalgebras, J. Pure Appl. Algebra 69 (1990), 111-120. MR 92b:17014

[KK] E. Kirkman and J. Kuzmanovich, Minimal prime ideals in enveloping algebras of Lie superalgebras, Proc. AMS 124 (1996), 1693-1702. MR 96h:16027

[L] E. S. Letzter, Prime and primitive ideals in enveloping algebras of solvable Lie superalgebras, Contempory Math. 130 (1992), 237-255. MR 93i:17003

[M] W. S. Martindale, III, Prime rings satisfying a generalized polynomial identity, J. Algebra 12 (1969), 576-584. MR 39:257

[P] D. S. Passman, Infinite Crossed Products, Academic Press, Boston, 1989. MR 90g:16002

[Pr] K. L. Price, Private communication, October, 1996.

[S] M. Scheunert, The Theory of Lie Superalgebras, Springer Lecture Notes in Mathematics, Vol. 716, Springer-Verlag, Berlin, 1979. MR 80i:17005

[Sm] L. W. Small, Orders in Artinian rings, J. Algebra 4 (1966), 13-41. MR 34:199

[W] M. C. Wilson, Delta methods in enveloping algebras of Lie colour algebras, J. Algebra 175 (1995), 661-696. MR 97a:17013

Department of Mathematics, DePaul University, Chicago, Illinois 60614

E-mail address: jbergen@condor.depaul.edu

Department of Mathematics, University of Wisconsin, Madison, Wisconsin 53706

E-mail address: passman@math.wisc.edu 